Research Article

\title{
Research on 2.4 GHz Wireless Channel Propagation Characteristics in a Steel Ship Cabin
}

\author{
Wanli Tu $\left(\mathbb{D},{ }^{1,2}\right.$ Hong Xu, ${ }^{1}$ Yiqun Xu, ${ }^{1,2}$ Qiubo Ye $\mathbb{D}^{3},{ }^{3}$ and Mingxian Shen ${ }^{4}$ \\ ${ }^{1}$ Marine Engineering Institute, Jimei University, Xiamen 361021, China \\ ${ }^{2}$ Fujian Provincial Key Laboratory of Naval Architecture and Marine Engineering, Xiamen 361021, China \\ ${ }^{3}$ Information Engineering Institute, Jimei University, Xiamen 361021, China \\ ${ }^{4}$ Navigation Institute, Jimei University, Xiamen 361021, China \\ Correspondence should be addressed to Wanli Tu; wanlitu@163.com
}

Received 21 October 2020; Revised 24 December 2020; Accepted 9 January 2021; Published 19 January 2021

Academic Editor: Atsushi Mase

Copyright (C 2021 Wanli Tu et al. This is an open access article distributed under the Creative Commons Attribution License, which permits unrestricted use, distribution, and reproduction in any medium, provided the original work is properly cited.

\begin{abstract}
Wireless sensor network (WSN) has become a popular technology and has a good potential application in naval architecture and marine engineering field. Analysis of transmission ability of wireless signal in steel ship environment is important for network deployment including base station and node installation, which would directly impact network performance and has become a hot research field. This paper investigates $2.4 \mathrm{GHz}$ frequency wireless signal propagation characteristics in the steel ship cabin. A 3D ray-tracing model of $2.4 \mathrm{GHz}$ wave propagation in the ship cabin is established, a multipath propagation prediction is carried on, and receiving power is calculated. Besides, the experimental test is performed in the real ship. The simulated and experimental results are processed and compared; the influence law of large obstacles on the signal is discussed, and the guidance scheme for node and base station deployment of the wireless sensor network is proposed. The results show that this analysis could provide valid interpretation of wireless channel propagation characteristics in steel ships.
\end{abstract}

\section{Introduction}

Wireless communication technology is becoming an important and promising mode for naval architecture and marine engineering field [1-3]. So far, more and more researchers focus on utilizing the wireless sensor network (WSN) for the application of marine environment monitoring and ship communication by the Internet of Things (IoT) and have made great progress [4-6]. Due to its significant advantages, the WSN has great prospects for smart ships' and unmanned ships' internal application. It will significantly reduce the ship manufacturing cost and production cycle compared to the cabled transmission method. It could also greatly reduce the fire risk which was often caused by the broken and aging cables. Besides, it has excellent advantage in the communication system renovation of the old ship. However, the most existing research studies are experimental tests focusing on the feasibility of wireless sensor work application in the ship so far and lack further analysis of improving the transmission performance of the wireless channel. Moreover, the network deployment is casual or mainly dependent on personnel experience, which would cause high cost and more time to build the network.

This paper investigates $2.4 \mathrm{GHz}$ frequency wireless signal transmission characteristics in various areas of the ship cabin, figures out favorable factors for stable signal transmission, and provides guidance for network deployment. Theoretical analysis by the ray-tracing model and experimental research in the real ship are both carried out, and the results were compared and discussed.

In Section 2, we motivate our approach by reviewing prior related research. Thereafter, we describe the raytracing method and subsequently propose the ray-tracing model of wireless signal propagation in the ship cabin. In Section 4, we present the experimental test in the real ship and compare the experimental results and simulated results. We then continue to do detailed discussion in Section 5 and provide concluding remarks in Section 6. 


\section{Related Work}

The research of WSN technology application on naval architecture began several years ago and has become a hot research topic. This section would introduce three aspects of the related state-of-the-art works in the literature: the research and development of WSN technology application on the ship cabin, the mythology to improve network performance, and the current theoretical analysis.

Earlier, Paik et al. employed $2.4 \mathrm{GHz}$ ZigBee communication technologies combined with the power line communication to establish the real-time monitoring system in a full-scale ship [7, 8]. Kdouh et al. validated the feasibility of ship WSN technology by measuring the radio wave propagation and proposed network topology based on hierarchical grouping for the large-scale ship to realize stable transmission in the steel cabin $[9,10]$. It provided an important foundation for the later development of ship WSN technology. Recently, lots of research studies have emerged and mainly worked for ship-related parameters monitoring, such as compartment environment and shipping container monitoring [11], vibration monitoring of the hull structure [12], fire early warning and monitoring for the ship bridge [13], and real-time status monitoring of the dynamic hazardous ship [14]. As can be seen, currently many ship WSN systems worked for small or simple-structured cabins with the form of the ad-hoc network. There are few research studies about WSN application in a more complex environment as well as for monitoring of large number of equipment on the ship.

There are several factors which would directly affect the communication quality of WSN, especially for complicated application, and have been getting attention from researchers. Some focused on the link reliability. Zhang et al. kept carrying out the investigation for performance optimization of WSN, including improving transmission performance by the combination of the quantum genetic strategy with the optimized-link state routing and improving the link reliability by the self-adaptive routing algorithm $[15,16]$. Some focused on the energy equilibrium of the WSN network. Liu et al. proposed novel unequal clustering routing protocols to balance the network energy consumption through network partition and distance [17]. Zhang et al. also proposed the energy-balanced routing method based on the forward-aware factor [18]. In addition, a few methods were proposed to improve other performance factors, e.g., improving recognition efficiency of nodes [19], the capacity of networks [20], and handling data exception or missing [21, 22].

On the contrary, simulation-based studies of wireless channel propagation in the ship would be greatly helpful for estimating the channel transmission characteristics and gaining the attention of researchers. Mariscotti et al. investigated the receiving power situation in the restaurant (large compartment) and corridor (narrow space) on board, and the results showed that the effect caused abundance of reflecting and diffracting elements which could not be ignored, and this method is unsuitable for the complex environments [23]. Ge et al. developed models to simulate radio wave transmission and attenuation in the small aisle space in the cabin by Maca ladder, and the results showed that the higher frequency will lead to more multipath effects [24]. Yan et al. established the ray-tracing based channel model and carried out propagation characteristics simulation of three communication frequencies including $900 \mathrm{MHz}, 2.4 \mathrm{GHz}$, and $60 \mathrm{GHz}$ in the ship corridor environment [25]. However, there is still a lack of theoretical analysis for wireless signal transmission in more complex environments such as the ship cabin.

Due to the ship's compact structure and complex environment, both simulation analysis and experimental test of wireless channel propagation characteristics would be necessary and important for establishing the WSN system in the steel ship. Thus, this paper focuses on the wireless wave propagation in the ship cabin based on the ray-tracing model and experimental test, which would be greatly helpful for deployment optimization of WSN.

\section{Ray-Tracing Model of Wireless Signal Propagation in the Ship Cabin}

When the wavelength is small compared with the geometry of an object, the propagation of electromagnetic waves can be approximated by geometric optics, and it is believed that electromagnetic waves travel along a straight line, following the laws of geometric optics. Reflection, penetration, and diffraction are three main ways when wireless signal faces obstacles. A large number of metal equipment in the ship structure would become the main factor affecting the wireless signal. It has a shielding effect on electromagnetic waves, which makes reflection and diffraction as obstacles for the wireless signal. It is difficult to calculate all the propagation paths between the two points within a complex structure. However, when ignoring those arrival paths with relatively small amplitude which have little effect on signal transmission stability, the propagation path can be identified with the ray-tracing method.

The ray-tracing method is based on geometrical optics (GO) and geometric uniform diffraction theory (UTD) and has high accuracy [26]. It was validated as an effective method for solving the problem of radio wave transmission planning and prediction in an indoor complex environment. The emitted electromagnetic wave propagates in all directions. According to geometrical position and electromagnetic parameter of obstacles, the propagation prediction can be determined by comprehensive analysis of reflection, transmission, and diffraction of each tracking ray. The principle of ray tracing is shown in Figure 1, where Tx is the transmitter and $\mathrm{Rx}$ is the receiver [27].

The received power is an important parameter to evaluate the communication system and characterize the received signal strength, which determines whether the receiving end can receive information well $[28,29]$. It is defined as follows: 


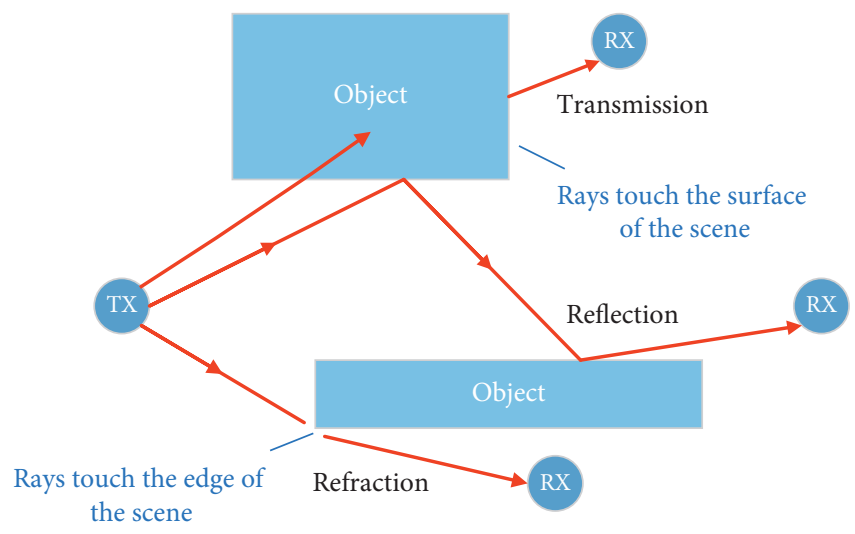

FIgURE 1: The principle of ray tracing.

$$
P_{N}=\sum_{i=1}^{N_{P}} P_{i}
$$

where $P_{N}$ is the average received power of total propagation path, $N_{P}$ is the number of propagation path, and $P_{i}$ is the average received power of the $i$ th propagation path, which is calculated as follows:

$$
P_{i}=\frac{\lambda^{2} \beta}{8 \pi \eta_{0}}\left|E_{\theta, i} g_{\theta}\left(\theta_{i}, \phi_{i}\right)+E_{\phi, i} g_{\phi}\left(\theta_{i}, \phi_{i}\right)\right|^{2},
$$

where $\lambda$ is signal wave length, $\eta_{0}$ is impedance of free space, and $E_{\theta, i}$ and $E_{\phi, i}$ are electric field intensity of the $i$ th receiving path in $\theta$ and $\phi$ directions, respectively. And,

$$
\begin{aligned}
& g_{\theta}\left(\theta_{i}, \phi_{i}\right)=\sqrt{\left|G_{\theta}\left(\theta_{i}, \phi_{i}\right)\right|} e^{j \psi_{\theta}}, \\
& g_{\phi}\left(\theta_{i}, \phi_{i}\right)=\sqrt{\left|G_{\phi}\left(\theta_{i}, \phi_{i}\right)\right|} e^{j \psi_{\phi}},
\end{aligned}
$$

where $\theta_{i}$ and $\phi_{i}$ are the arrival direction of the $i$ th ray, $G_{\theta}$ and $G_{\phi}$ are antenna gain of the receiver in $\theta$ and $\phi$ directions, respectively, and $\psi_{\theta}$ and $\psi_{\phi}$ are phases in $\theta$ and $\phi$ directions of the far zone, respectively.

Besides, $\beta$ is defined as follows:

$$
\beta=\frac{\int_{f_{T}-\left(B_{T} / 2\right)}^{f_{T}+\left(B_{T} / 2\right)} S_{T}(f) S_{R}(f) \mathrm{d} f}{\int_{f_{T}-\left(B_{T} / 2\right)}^{f_{T}+\left(B_{T} / 2\right)} S_{T}(f) \mathrm{d} f},
$$

where $f_{T}$ and $B_{T}$ are center frequency and bandwidth of the transmitter and $S_{T}(f)$ and $S_{R}(f)$ are the spectrum of the transmitter and receiver, respectively. Usually, the narrow band wave spectrum is assumed as flat distribution:

$$
S(f)= \begin{cases}1, & f_{0}-\frac{B}{2}<f<f_{0}+\frac{B}{2}, \\ 0, & \text { others, }\end{cases}
$$

where $f_{0}$ and $B$ are center frequency and bandwidth of the narrow band wave [30].
All simulations reported here are performed based on the ray-tracing method, using the Remcom Wireless InSite commercial software package.

The wireless signal propagation in the ship cabin is investigated. The large-scale sand carrier "Zhaoming Wheel" is adopted for the research subject. Figure 2 shows the structure of the "Zhaoming Wheel" and three-dimensional model.

Figure 2(a) is the sketch of ship structure "Zhaoming Wheel," Figure 2(b) is the three-dimensional model of the ship cabin, and Figures 2(c) and 2(d) are the second floor of the engine room and platform of the control room, respectively. The total length of the ship is $169 \mathrm{~m}$, the moulded depth is $13.6 \mathrm{~m}$, and the maximum height is 43 meters. It includes the main engine room, control room, accommodation area, and staircase connecting the platforms. The three-dimensional model of the ship cabin is established by UG8.0 3D modeling software and is saved as the STL file format. Then, it is imported into Wireless InSite.

The material of the structure and equipment is set as steel. The door of the control room as well as other doors between floors are made of a fireproof material. The windows of the control room are made of glass, and the chairs are made of wood. All doors in the cabin are open, and the window is closed. An omnidirectional monopole antenna with length $30 \mathrm{~mm}$, working in the vertical polarization mode, is chosen in this investigation because the vertical polarization mode leads to lower path loss than the horizontal polarization mode and has the best coverage in the indoor environment [31]. The performance is investigated in the center frequency of $2400 \mathrm{MHz}$, with the bandwidth $2 \mathrm{MHz}$, the path gain $15 \mathrm{dBi}$, and the transmitting signal adopts the sinusoid wave shape. The other simulation parameters are shown Table 1.

Furthermore, in all simulations, the factors such as atmospheric propagation losses, nonuniformities of the structure surface, and influence of small objects such as thin pipes on signal transmission are not considered for simplicity, as these produce little contribution to the total receiving power. The transmitter and receiver nodes are deployed, which are shown in Figure 3. Figures 3(a) and 3(b) are the side view and vertical view of the node deployment in the ship cabin.

The initial transmitter $(\mathrm{Tx})$ position is set at the bottom platform of the main engine room. The receivers $(\mathrm{Rx})$ are located at various areas including the bottom floor of the cabin ( $\mathrm{Rx} 1, \mathrm{Rx} 2)$, center of the control room ( $\mathrm{Rx} 3)$, first floor of accommodation $(\mathrm{Rx} 4)$, restaurant on the second floor of accommodation ( $\mathrm{Rx} 5)$, wheel house ( $\mathrm{Rx} 6)$, and middle part of the weather deck $(\operatorname{Rx} 7)$. The simulated results of the receiving power, path loss etc. are obtained for further analysis. In the bottom platform of the main engine room, receivers $\mathrm{Rx} 1$ and $\mathrm{Rx} 2$ are located on the floor and beside the main engine. The receivers are not far from the transmitter $\mathrm{Tx}$, but both of them are in the non-line-of-sight range. As shown in Figure 3(b), Rx1 is set diagonally with Tx, and Rx2 is placed at the edge on the other side, which aims to 


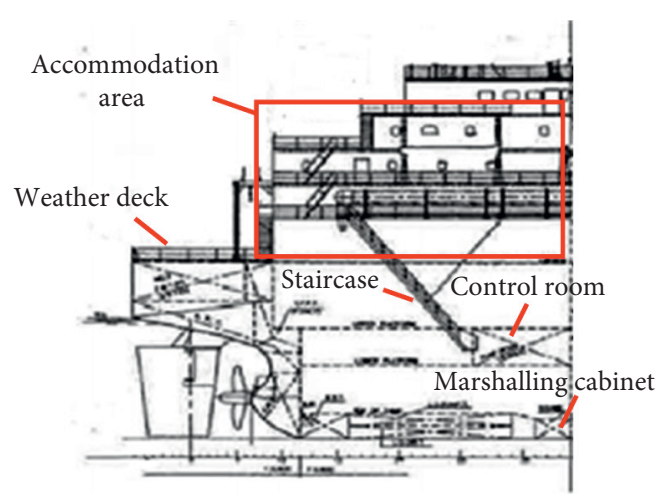

(a)

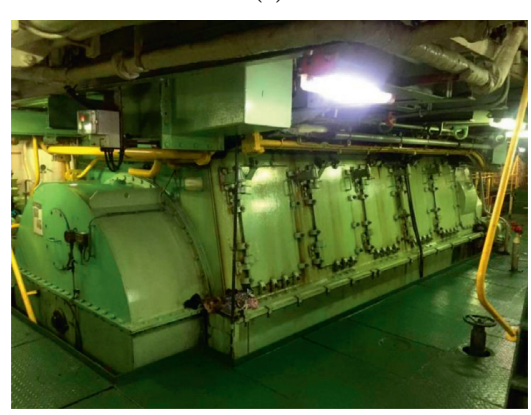

(c)

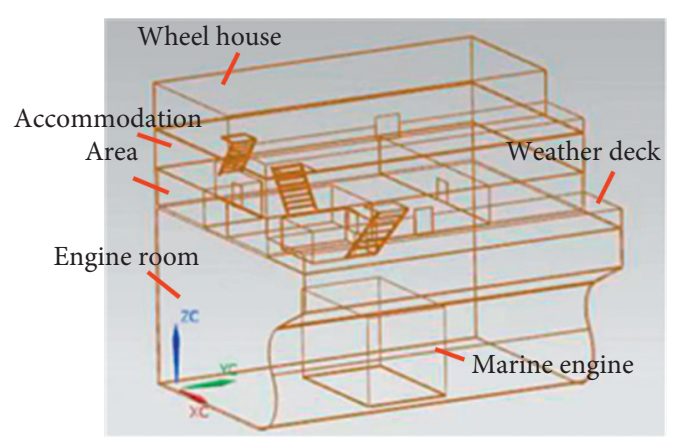

(b)

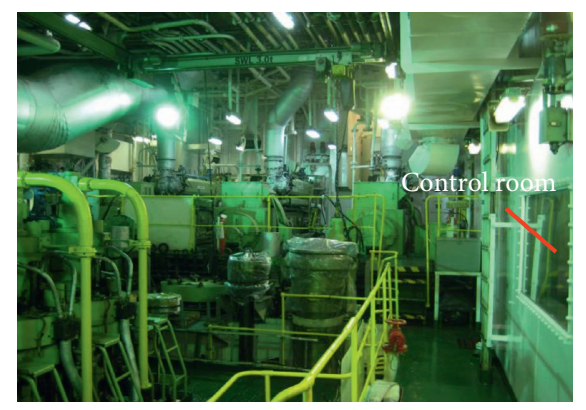

(d)

FIgURE 2: (a) Sketch of the ship structure "Zhaoming Wheel." (b) The three-dimensional model of the ship cabin. (c) The second floor of the engine room. (d) The platform of the control room.

TABLE 1: The simulation parameter.

\begin{tabular}{lc}
\hline Parameter & Setting value \\
\hline Transmit model & X3D \\
Waveform & Sinusoid \\
Carrier frequency & $2400 \mathrm{MHz}$ \\
Effective bandwidth & $2 \mathrm{MHz}$ \\
Transmitter & Linear monopole omnidirectional antenna \\
Receiver & Linear monopole omnidirectional antenna \\
Antenna gain & $15 \mathrm{dBi}$ \\
Ray interval & 0.25 \\
Reflections & 5 \\
Transmission & 2 \\
Refraction & 1 \\
Received rays & 20 \\
\hline
\end{tabular}

investigate signal transmission when large obstacle exists. Both Rx1 and Rx2 have good reception; the received power are $-40.17 \mathrm{dBm}$ and $-33.24 \mathrm{dBm}$, respectively. Figure 4 shows their effective receiving paths with large energy. Obviously, the $\mathrm{Rx} 2$ has larger receiving strength and smaller path loss than Rx1. There are few reflections of signals emitted from Tx to $\mathrm{Rx} 2$, and diffraction results in less signal attenuation. In contrast, due to the main engine as the large obstacle in the middle of the diagonal, signals emitted from TX to Rx1 need multiple reflections and have large signal attenuation.

The central control room is located in the middle platform of the main engine room, and corridor and pipes around the engine and auxiliary machine are located at the tail area. There is a door and a glass window with
$1.2 \mathrm{~m} \times 0.8 \mathrm{~m}$ size in the control room, and the main power distribution board, chairs, and air conditioners are large obstacles. The other structures are simplified. The receiver $\mathrm{Rx} 3$ is set in the middle of the control room, with 9 nodes (\#1 \#9) forming a $3.5 \mathrm{~m} \times 3.5 \mathrm{~m}$ size square deployment, as shown in Figure 5(a). Although there is partition between the receiver and transmitter on the bottom platform, the signal transmission does not get severe interference due to existence of the door and window. The farther the receiver from the hatch door, the lower the received power. The results show the maximum receiving power $-39.4 \mathrm{dBm}$ occurring at the \#7 node. Figure 5(b) shows the three paths with the strongest signal strength. The signals emitted from Tx occur reflecting on the metal wall of the cabin and reach Rx3 through the control room door.

The living accommodation area is on the top of the main engine room and includes the activity room on the first floor and the restaurant on the second floor. Considering the long distance between the transmitter and receiver, the receivers were placed at the exit of the connecting staircases. The receiver $\mathrm{Rx} 4$ was with 15 nodes (\#1 \#15) forming a $3.5 \mathrm{~m} \times 3.5 \mathrm{~m}$ size square deployment, as shown in Figure 6(a). The results show that although the activity room is far from the $\mathrm{Tx}$, the path loss is not great because the staircase provides the transmission channel. The maximum receiving power occurring at the \#4 node is $-34.3 \mathrm{dBm}$, which is even higher than the maximum value in the control room below. Figure 6(b) shows a few transmission paths of the \#4 node, where some wireless signal is reflected only once and then transmitted directly to the receiving node by 


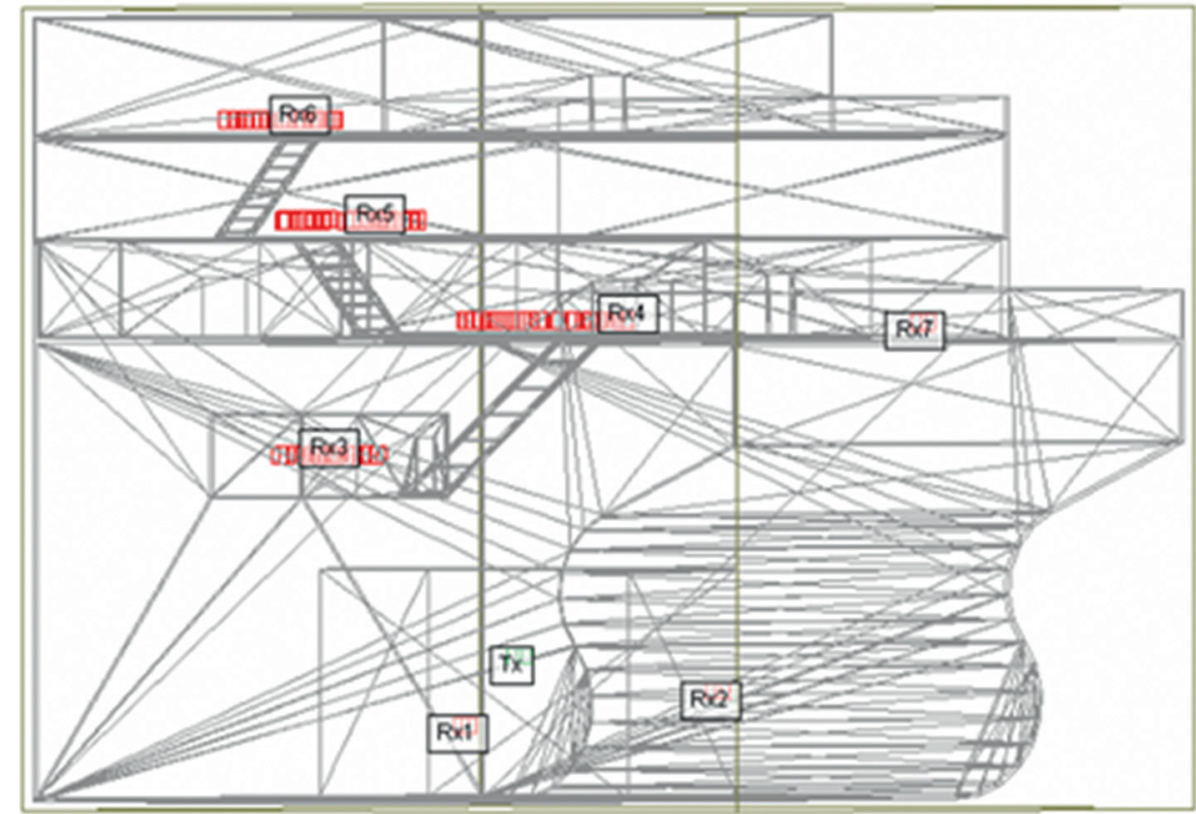

(a)

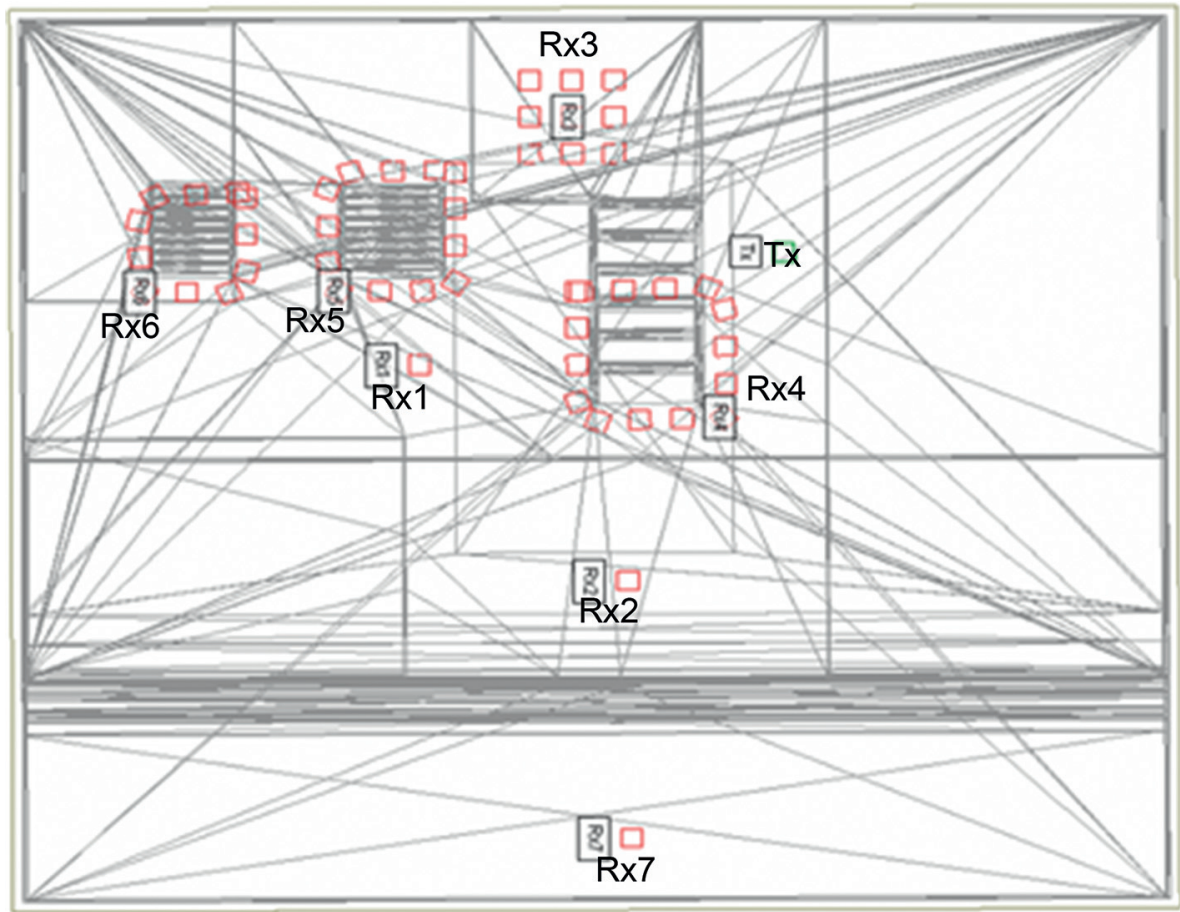

(b)

Figure 3: Deployment of the transmitter and receiver in the ship cabin. (a) The side view. (b) The vertical view.

the staircase. The less frequent reflections significantly reduce the loss of receiving power. Few reflections cause little signal energy loss.

When it gets to the upper layer, that is, the second floor of accommodation, the situation of the receiver Rx5 is worse. The receiver Rx5 was with 13 nodes (\#1 \#13) forming a $3.2 \mathrm{~m} \times 3 \mathrm{~m}$ size rectangular deployment, as shown in Figure 7(a). Several nodes have a very low value of received power. As shown in Figure 7(b), no reception occurs at the
\#4 and \#13 nodes, the effective signal transmission paths of $\# 1 \sim \# 7$ nodes (excluding \#4) are significantly reduced, and some nodes in \#8 \#12 could not reach the preset number 20. From the nodes position, it can be found that \#1 \#7 and \#13 nodes are located on the same side of Tx. In contrast, \#8 \#12 nodes are on the opposite side of Tx. The signals emitted from Tx need to pass through the little component beside the staircase connecting the first floor and second floor of live accommodation and then reach the 


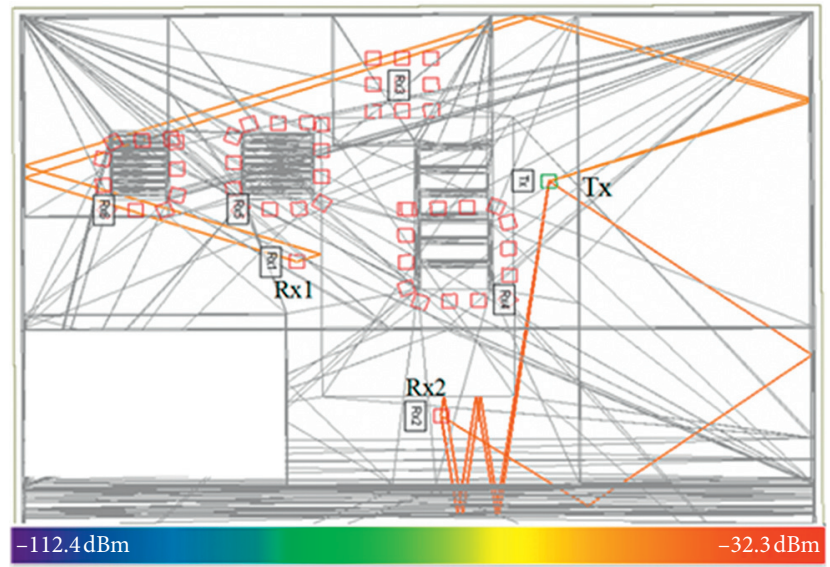

FiguRE 4: Simulated result of $\mathrm{Rx} 1$ and $\mathrm{Rx} 2$ receivers with several effective receiving paths.
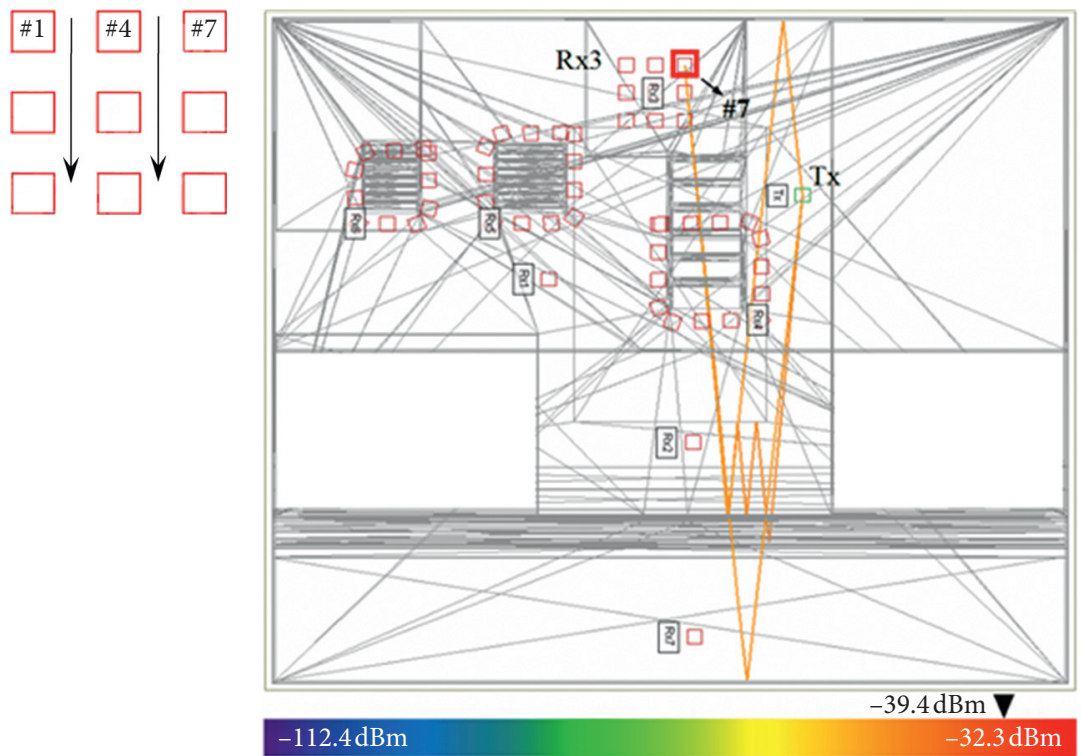

(a)

(b)

Figure 5: Simulated results of the Rx3 receiver. (a) Node deployment of Rx3. (b) Three paths with the strongest signal strength of Rx3.

\#1 \#7 nodes. The reflection increases which would result in large signal attenuation, compared to the nodes on the other side. The maximum receiving power $-54.9 \mathrm{dBm}$ occurred at the $\# 9$ node. Figure 7 (c) shows the paths with the strongest signal strength, where three paths transmit directly to the \#9 node by the staircase connection to the control room and first floor of the accommodation area after few reflections.

The wheel house is on the highest platform of the ship, which is far away from the engine room. The receiver Rx6 is place on the floor of the wheel house, with 11 nodes (\#1 \#11) forming a $3 \mathrm{~m} \times 2.5 \mathrm{~m}$ size rectangular deployment, as shown in Figure $8(\mathrm{a})$. The reception is bad, where only \#8 and \#9 nodes have few effective transmission paths, as shown in Figure 8(b). However, the maximum receiving power $-79.4 \mathrm{dBm}$ occurred at the $\# 8$ node. The signals emitted from Tx generally cause multiple reflections by the staircases or railings before they reach the receiver in the wheel house, as shown in Figures 8(c) and 8(d). It is obvious that the hollow staircase could provide good aid for signal transmission.

The receiver $\mathrm{Rx} 7$ placed on the floor of weather deck has no reception. Although the weather deck is located at the same platform with the first floor, several crew cabins between the weather deck and staircase cause multiple reflections on the signal and large attenuation, failing to achieve the receive sensitivity.

\section{Experimental Test in Real Ship}

Experimental test carried out on the real ship is also necessary and important to analyze the wireless signal transmission, especially for a complex environmental scenario. On "Zhaoming Wheel," the experimental system adopts communication nodes with antenna and power supply. The transmitters and receivers are installed at 

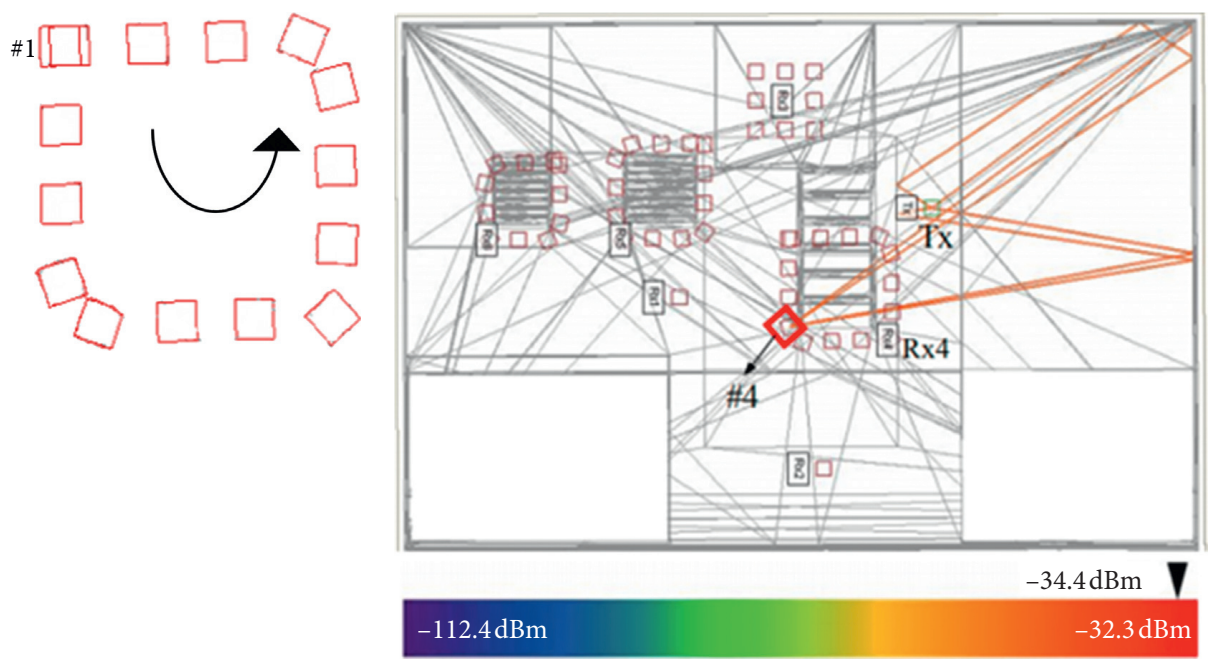

(a)

(b)

Figure 6: Simulated results of the Rx4 receiver. (a) Node deployment of Rx4. (b) A few transmission paths of the \#4 node of Rx4.

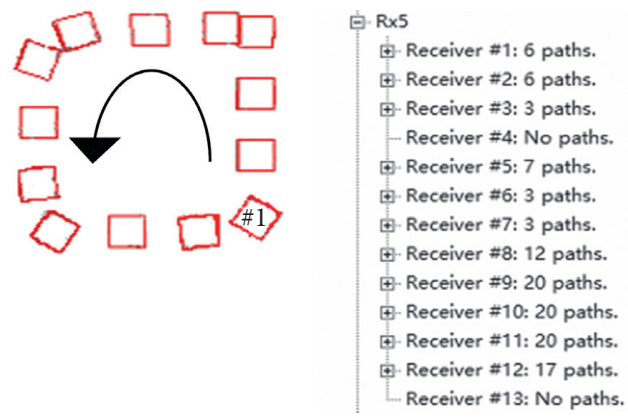

(a) (b)

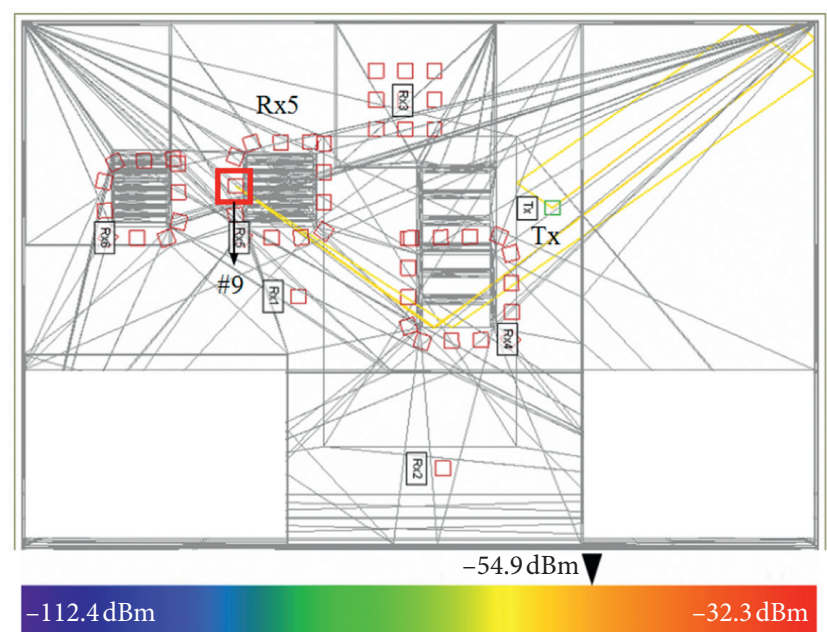

(c)

FIgURe 7: Simulated results of the Rx5 receiver. (a) Node deployment of Rx5. (b) Effective reception path situation of Rx5. (c) A few transmission paths of the \#9 node $\mathrm{Rx} 5$.

different positions, and the notebook computer is used to process the received power for further analysis, as shown in Figure 9. The experimental results of various areas are shown in Table 2. Besides, Figures 10(a)-10(c) show the receiving power comparison of simulated and experimental results of some nodes of $\mathrm{Rx} 3, \mathrm{Rx} 4$, and $\mathrm{Rx} 5$ receivers, respectively.

The results show that although there is only a wireless transmitter installed on the floor of the main engine cabin, several areas are able to receive the signal. These situations are similar for simulated results and experimental results. The main engine room, control room, and first floor of accommodation have good reception. The second floor of accommodation has good reception with few data losses. And, the wheel house and weather deck have bad reception. The received power of $\mathrm{Rx} 1$ and $\mathrm{Rx} 2$ placed in the main engine room are $-27.37 \mathrm{dBm}$ and $-34.38 \mathrm{dBm}$, respectively. The received power range of $\mathrm{Rx} 3$ in the control room is $-42.46 \mathrm{dBm} \sim-24.76 \mathrm{dBm}$, with the average as $-32.26 \mathrm{dBm}$; the received power range of $\mathrm{Rx} 4$ in the first floor of accommodation is $-61.79 \mathrm{dBm} \sim-37.32 \mathrm{dBm}$, with the average as $-47.13 \mathrm{dBm}$. When processing the results of the $\mathrm{Rx} 5$ receiver in the second floor of accommodation, the \#4 and \#13 nodes in simulation as well as the \#13 node in the experiment have no reception. Thus, $-150 \mathrm{dBm}$ is used to represent this situation when drawing the curve. Except the \#13 node, the experimental received power range of $\mathrm{Rx} 5$ is $-68.13 \mathrm{dBm} \sim-221257.16 \mathrm{dBm}$, with the average as $-62.92 \mathrm{dBm}$.

It should be noted that the antenna was placed in a relatively simple way due to experimental conditions. The experimental results obtained by the point-to-point test here 

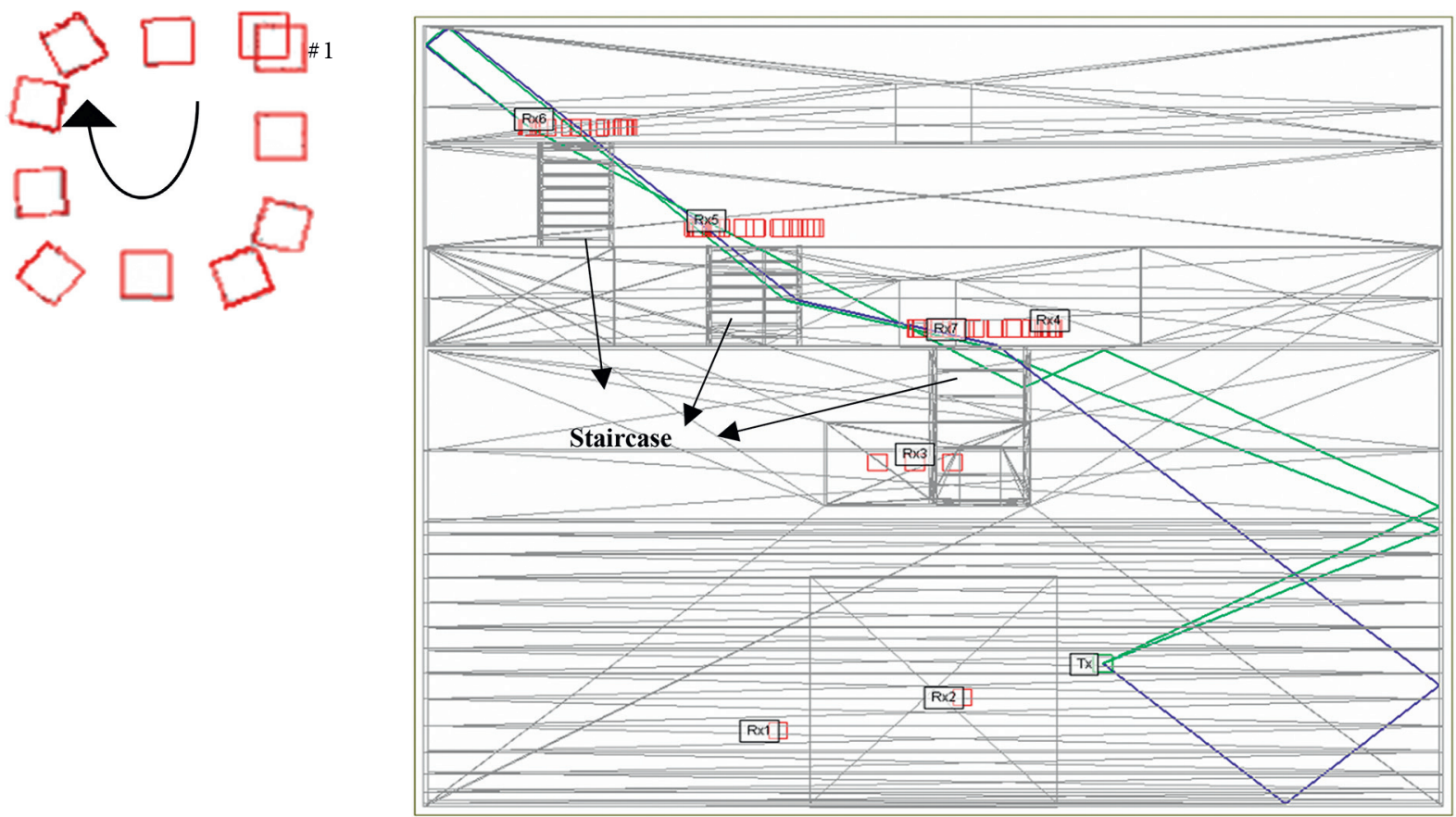

(a)

(b)

巨. Rx6

\author{
Receiver \#1: No paths. \\ Receiver \#2: No paths. \\ Receiver $\# 3$ : No paths. \\ Receiver \#4: No paths. \\ Receiver $\# 5$ : No paths. \\ Receiver \#6: No paths. \\ Receiver $\# 7$ : No paths. \\ 7. Receiver \#8: 4 paths. \\ \$. Receiver \#9: 1 paths. \\ Receiver $\# 10$ : No paths. \\ Receiver $\# 11$ : No paths.
}

(c)

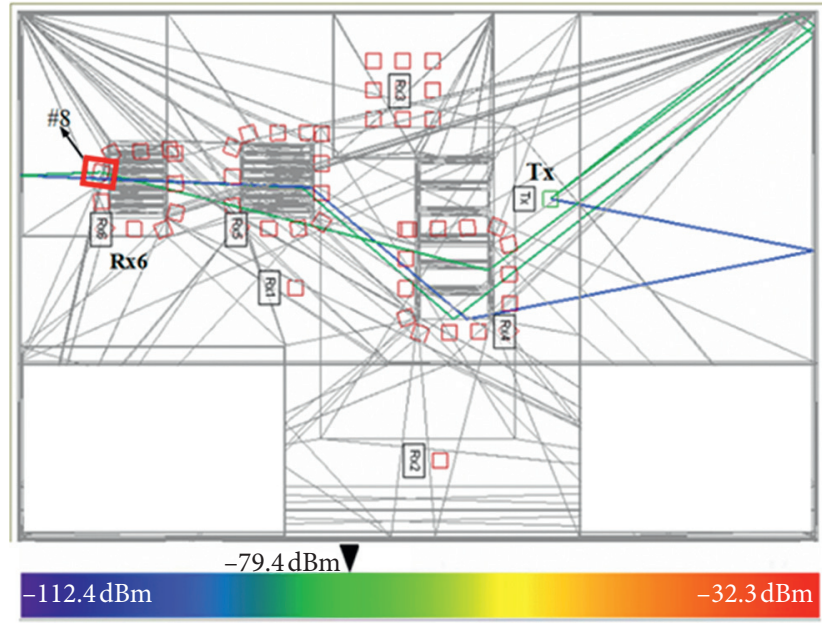

(d)

FIgURE 8: Simulated results of the Rx6 receiver. (a) Node deployment of Rx6. (b) The effective reception path situation of Rx6. (c) The side view of transmission paths of the \#8 node Rx6. (d) The vertical view transmission paths of the \#8 node Rx6.

is effective when comparing with the simulated results. However, when establishing the WSN system in the future work, the better positioning method would be adopted to avoid surrounding elements that introduce additional distortions to the signal [32].

\section{Discussion}

Although the experimental point-to-point test results showed that the proposed method could provide valid interpretation of wireless channel propagation, differences existing between simulated and experimental results cannot be ignored. The values of receiving power are obviously different, and reception performance of some nodes is totally contrasting. It is mainly because of the model error where the model could not fully represent the actual ship structure. Besides, the electromagnetic field interference and noise of the equipment in the ship cabin may be other contributing factors. Even so, when predicting the signal propagation characteristics and reception of various regions of the ship cabin, the simulated results are agreed well with the situation of experimental results. It verifies that the ray-tracing model can play an important guidance role for WSN establishment. Combining the ray-tracing simulation with experimental 


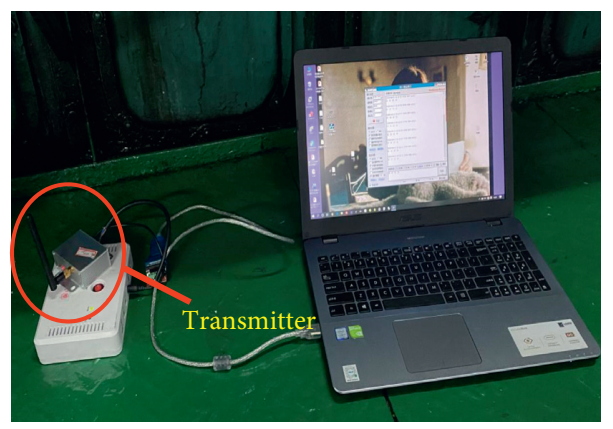

(a)

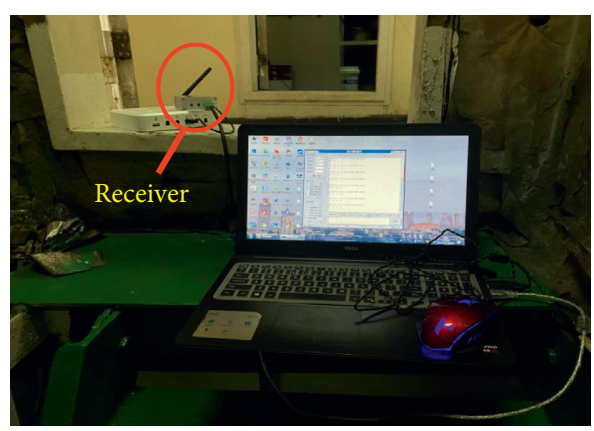

(b)

FIgURE 9: (a) The transmitter of the experimental system. (b) The receiver of the experimental system.

TABLe 2: Experimental results of the "Zhaoming" wheel.

Receiver

Main engine room cabin $(\mathrm{Rx} 1, \mathrm{Rx} 2)$

Control room $(\mathrm{Rx} 3)$

First floor of accommodation $(\mathrm{Rx} 4)$

Second floor of accommodation (Rx5)

Wheel house (Rx6)

Weather deck (Rx7)
Receiving performance

Good reception

Good reception

Good reception

Good reception and few data loss

Poor reception and serious data loss No reception
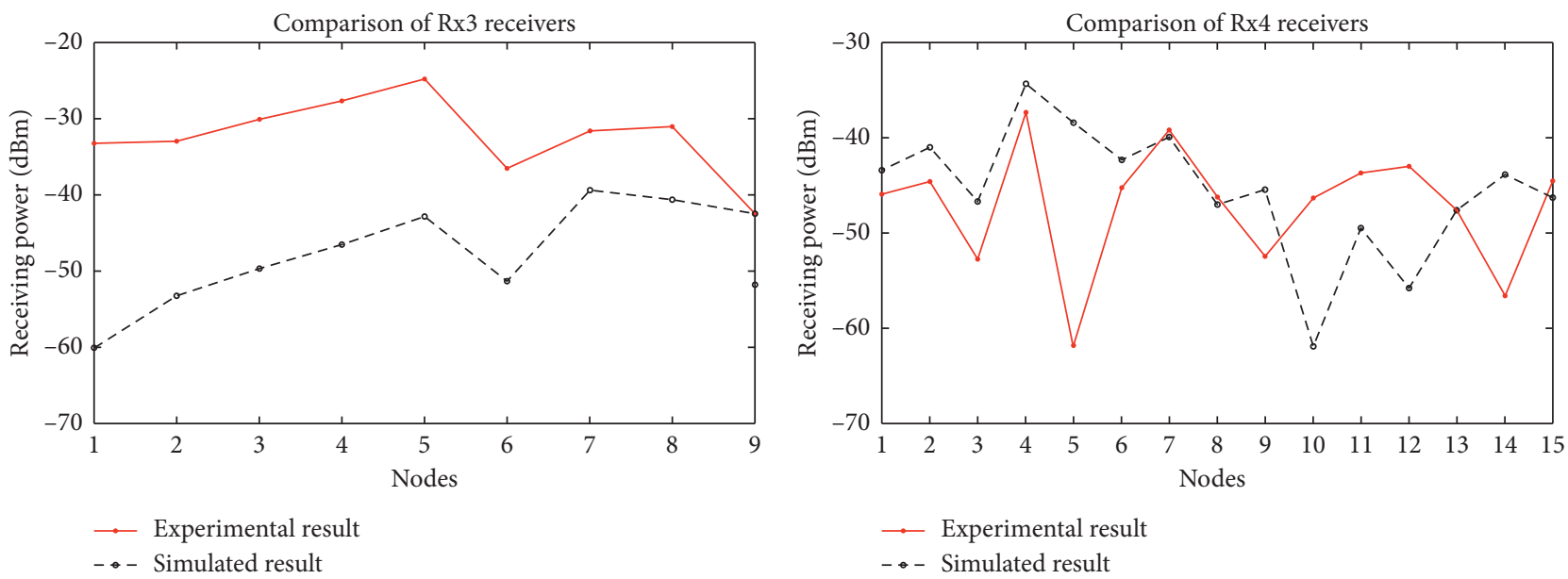

(a)

(b)

FIgUre 10: Continued. 


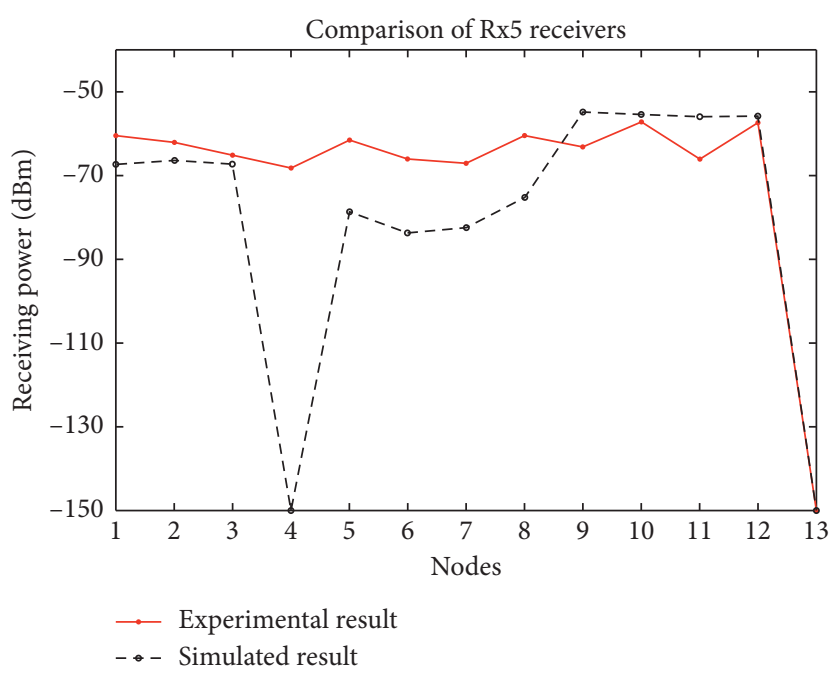

(c)

FIGURE 10: Comparison of simulated and experimental results. (a) Receiving power of nodes of Rx3. (b) Receiving power of nodes of Rx4. (c) Receiving power of nodes of Rx5.

test on the real ship could narrow down the deployment scope of network nodes and reduce the time. Some guidance scheme can be drawn as follows:

(i) From the simulation and experiment analysis, it verifies the diffraction ability of the $2.4 \mathrm{GHz}$ frequency band wave when it encounters the large equipment obstacle. However, large obstacles could still cause a great blocking effect on the wireless signal, especially when the nodes are placed in the opposite position of the obstacle. When the wireless signal propagates in the main engine cabin, the network nodes should be set at the location of relatively empty area with less obstacles. On the contrary, adjusting the node bested the edge of the large obstacle which could reduce reflections, increase the receiving power, and improve transmission quality.

(ii) When it comes to the complex environment, simulation analysis can be initially used to predict and analyze the favorable node location and signal channel, and the on-site measurement and commission is also important for final confirmation.

(iii) Nonmetallic partition (such as doors and windows) provided good channels for wireless signal transmitting in the ship environment. The nodes of WSN can be arranged near the windows or doors. Another good aid for signal transmission is the hollow staircase which is widely distributed in the ship cabin. The routes or base stations of WSN can be deployed in these locations to assure a relatively steady transmission path.

(iv) Due to the random effect caused by the complex environment, the obvious fluctuation existed with wireless signal transmission in the higher platforms. It could be resolved by deploying the wireless communication microbase station on each platform, which is used to connect the wireless communication nodes on the same platform. Besides, due to the short distance of the $2.4 \mathrm{GHz}$ frequency band, the multirelay could be appropriately applied to achieve the ideal transmission effect in the ship cabin. Then, the microbase station can be connected to the onboard legacy (wired) infrastructure, which could assure a highly reliable WSN system.

\section{Conclusion}

Compared with the analog signal system, the digital signal system has higher requirement of the communication channel. Unreasonable network deployment could cause large loss of signal transmission, resulting in abnormal communication. Although the ship's structural environment is complex, it is not seriously a changing scenario. The design of the ship cabin, main engine, staircase, weather deck, etc., is generally followed in shipbuilding specifications. In this paper, the $2.4 \mathrm{GHz}$ frequency band wave propagating in the ship cabin is analyzed in detail based on the ray-tracing model and experimental test. Most simulated results are with good consistency with the experimental results, especially in the reception estimation of various regions of the ship cabin and propagation prediction of the signal. The simulation analysis based on the ray-tracing method would be greatly helpful for WSN design and node deployment in the ship.

\section{Data Availability}

All the data included in this study are available from the corresponding author upon request. 


\section{Conflicts of Interest}

The authors declare that they have no conflicts of interest regarding the publication of this paper.

\section{Acknowledgments}

The authors gratefully acknowledge the support from the Regional Demonstration Project of Marine Economic Innovation and Development in Fujian Province, State Oceanic Administration (subtopicB17090), Fujian Provincial Natural Science Foundation (Grant no. 2020J01683), and Jimei University National Fund Breeding Program (Grant no. 2P2020012), as well as the large-scale sand carrier "Zhaoming Wheel" which provided help for experimental measurement of this paper.

\section{References}

[1] J. Molka-Danielsen, P. Engelseth, and H. Wang, "Large scale integration of wireless sensor network technologies for air quality monitoring at a logistics shipping base," Journal of Industrial Information Integration, vol. 10, pp. 20-28, 2018.

[2] J. Yang, C. Wang, Q. Zhao, B. Jiang, Z. Lv, and A. K. Sangaiah, "Marine surveying and mapping system based on cloud computing and internet of things," Future Generation Computer Systems, vol. 85, pp. 39-50, 2018.

[3] S. S. Bennett, C. J. Brooks, B. Winden et al., "Measurement of ship hydroelastic response using multiple wireless sensor nodes," Ocean Engineering, vol. 79, pp. 67-80, 2014.

[4] P. David, "Enabling the maritime internet of things: CoAP and 6LoWPAN performance over VHF links," IEEE Internet of Things Journal, vol. 5, no. 6, pp. 5205-5212, 2018.

[5] H. Zhang and X. Lu, "Vehicle communication network in intelligent transportation system based on internet of things," Computer Communications, vol. 160, pp. 799-806, 2020.

[6] R. Al-Zaidi, J. C. Woods, M. Al-Khalidi, and H. Hu, "Building novel VHF-based wireless sensor networks for the internet of marine things," IEEE Sensors Journal, vol. 18, no. 5, pp. 2131-2144, 2018.

[7] B.-G. Paik, S.-R. Cho, B.-J. Park, D. Lee, B.-D. Bae, and J.-H. Yun, "Characteristics of wireless sensor network for fullscale ship application," Journal of Marine Science and Technology, vol. 14, no. 1, pp. 115-126, 2009.

[8] B.-G. Paik, S.-R. Cho, B.-J. Park, D. Lee, and B.-D. Bae, "Development of real-time monitoring system using wired and wireless networks in a full-scale ship," International Journal of Naval Architecture and Ocean Engineering, vol. 2, no. 3, pp. 132-138, 2010.

[9] H. Kdouh, H. Farhat, G. Zaharia, C. Brousseau, G. Grunfelder, and G. E. Zein, "Performance analysis of a hierarchical shipboard wireless sensor network," in Proceedings of the IEEE 23rd International Symposium on Personal Indoor and Mobile Radio Communications (PIMRC), Sydney, Australia, September 2012.

[10] H. Kdouh, G. Zaharia, C. Brousseau, G. Grunfelder, H. Farhat, and G. E. Zein, "Wireless sensor network on board vessels," in Proceedings of the 19th International Conference on Telecommunications, Jounieh, Lebanon, April 2012.

[11] X. Yang and Y. Zhang, "Location algorithm for nodes of shipborne wireless sensor networks," International Journal of Distributed Sensor Networks, vol. 8, pp. 1172-1175, 2013.
[12] X. Y. Xiong, F. Wei, J. W. Li, M. Han, and D. H. Guan, "Vibration monitoring system of ships using wireless sensor networks," in Proceedings of the 2014 IEEE International Conference on Mechatronics and Automation, Tianjin, China, August 2014.

[13] S. B. Wu, X. Q. Chen, H. X. Chen, and J. Q. Lu, "Intelligent fire early warning and monitoring system for ship bridge based on WSN," International Journal of Science, vol. 7, no. 8, pp. $248-255,2020$.

[14] Y. Ma, K. Liu, M. Chen et al., "Deadline-aware adaptive emergency navigation strategy for dynamic hazardous ship evacuation with wireless sensor networks," IEEE Access, vol. 99, pp. 135758-135769, 2020.

[15] D.-G. Zhang, T. Zhang, Y. Dong, X.-H. Liu, Y.-Y. Cui, and D.-X. Zhao, "Novel optimized link state routing protocol based on quantum genetic strategy for mobile learning," Journal of Network and Computer Applications, vol. 122, pp. 37-49, 2018.

[16] D. Zhang, T. Zhang, and X. Liu, "Novel self-adaptive routing service algorithm for application in VANET," Applied Intelligence, vol. 49, no. 5, pp. 1866-1879, 2019.

[17] D. G. Zhang, S. Liu, T. Zhang, and Z. Liang, "Novel unequal clustering routing protocol considering energy balancing based on network partition \& distance for mobile education," Journal of Network and Computer Applications, vol. 88, pp. 1-9, 2017.

[18] D. Zhang, G. Li, K. Zheng, X. Ming, and Z.-H. Pan, “An energy-balanced routing method based on forward-aware factor for wireless sensor networks," IEEE Transactions on Industrial Informatics, vol. 10, no. 1, pp. 766-773, 2014.

[19] D. Zhang, X. Wang, X. Song, and D. Zhao, "A novel approach to mapped correlation of ID for RFID anti-collision," IEEE Transactions on Services Computing, vol. 7, no. 4, pp. 741-748, 2014.

[20] J. Chen, G. Mao, C. Li, W. Liang, and D.-G. Zhang, "Capacity of cooperative vehicular networks with infrastructure support: multiuser case," IEEE Transactions on Vehicular Technology, vol. 67, no. 2, pp. 1546-1560, 2018.

[21] T. Zhang, D.-G. Zhang, H.-R. Yan, J.-N. Qiu, and J.-X. Gao, "A new method of data missing estimation with FNN-based tensor heterogeneous ensemble learning for internet of vehicle," Neurocomputing, vol. 420, no. 1, pp. 98-110, 2021.

[22] L. Li, "Real time auxiliary data mining method for wireless communication mechanism optimization based on internet of things system," Computer Communications, vol. 160, pp. 333-341, 2020.

[23] A. Mariscotti, "Experimental determination of the propagation of wireless signals on board a cruise ship," Measurement, vol. 44, no. 4, pp. 743-749, 2011.

[24] Y. M. Ge and J. Li, "Simulation and analysis of radio wave propagation characteristic in cabin," Journal of Sichuan Ordnance, vol. 9, pp. 92-95, 2014, in Chinese.

[25] C. Yan, L. H. Ge, and X. P. Fan, "Simulation and analysis of radio wave propagation characteristics in ship cabin," Journal of Shanxi Datong University (Natural Science), vol. 34, no. 6, pp. 17-20, 2018, in Chinese.

[26] G. R. Maccartney, T. S. Rappaport, S. Sun, and S. Deng, "Indoor office wideband millimeter-wave propagation measurements and channel models at 28 and $73 \mathrm{GHz}$ for ultra-dense 5G wireless networks," IEEE Access, vol. 3, pp. 2388-2424, 2015.

[27] X. Xu, Z. Zhang, Y. Xu et al., "Measurement and analysis of wireless propagative model of $433 \mathrm{MHz}$ and $2.4 \mathrm{GHz}$ 
frequency in Southern China orchards," IFAC-PapersOnLine, vol. 51, no. 17, pp. 695-699, 2018.

[28] S. Shikhantsoy, A. Thielens, G. Vermeeren et al., "Industrial indoor massive MIMO human EM-exposure evaluation," in Proceedings of the Joint Annual Meeting of the Bio Electromagnetics Society and the European Bio Electromagnetics Association, pp. 403-406, Montpellier, France, June 2018.

[29] P. Labbé, J. Gosteau, M. Muck, J. Pamp, and R. Baggen, "Impact of macro diversity on the final throughput of a $60 \mathrm{GHz}$ SFN deployment," in Proceedings of the 14th IST Mobile \& Wireless Communications Summit, Dresden, Germany, June 2005.

[30] 3D Wireless Communication and Propagation Prediction Software Reference Manual Version 3.2.0, 2017, http://www. remcom.com/.

[31] G. H. Xu, G. J. Su, H. L. Wang, Q. G. Wang, and Y. Liu, "Analyzing effects of antenna on indoor ZigBee channel transmission characteristics," Computer Science, vol. 43, no. 4, pp. 92-96, 2016, in Chinese.

[32] J. Yang, M. Ding, G. Mao, Z. Lin, D.-G. Zhang, and T. H. Luan, "Optimal base station antenna downtilt in downlink cellular networks," IEEE Transactions on Wireless Communications, vol. 18, no. 3, pp. 1779-1791, 2019. 